Document downloaded from:

http://hdl.handle.net/10251/65275

This paper must be cited as:

Garcia Hernandez, CR.; Rosales, J.; García Fajardo, L.; Pérez-Navarro Gómez, A.; Escrivá, A.; Abánades, A. (2011). Performance of a transmutation advanced device for sustainable energy application. Progress in Nuclear Energy. (53):1151-1158.

doi:10.1016/j.pnucene.2011.07.009

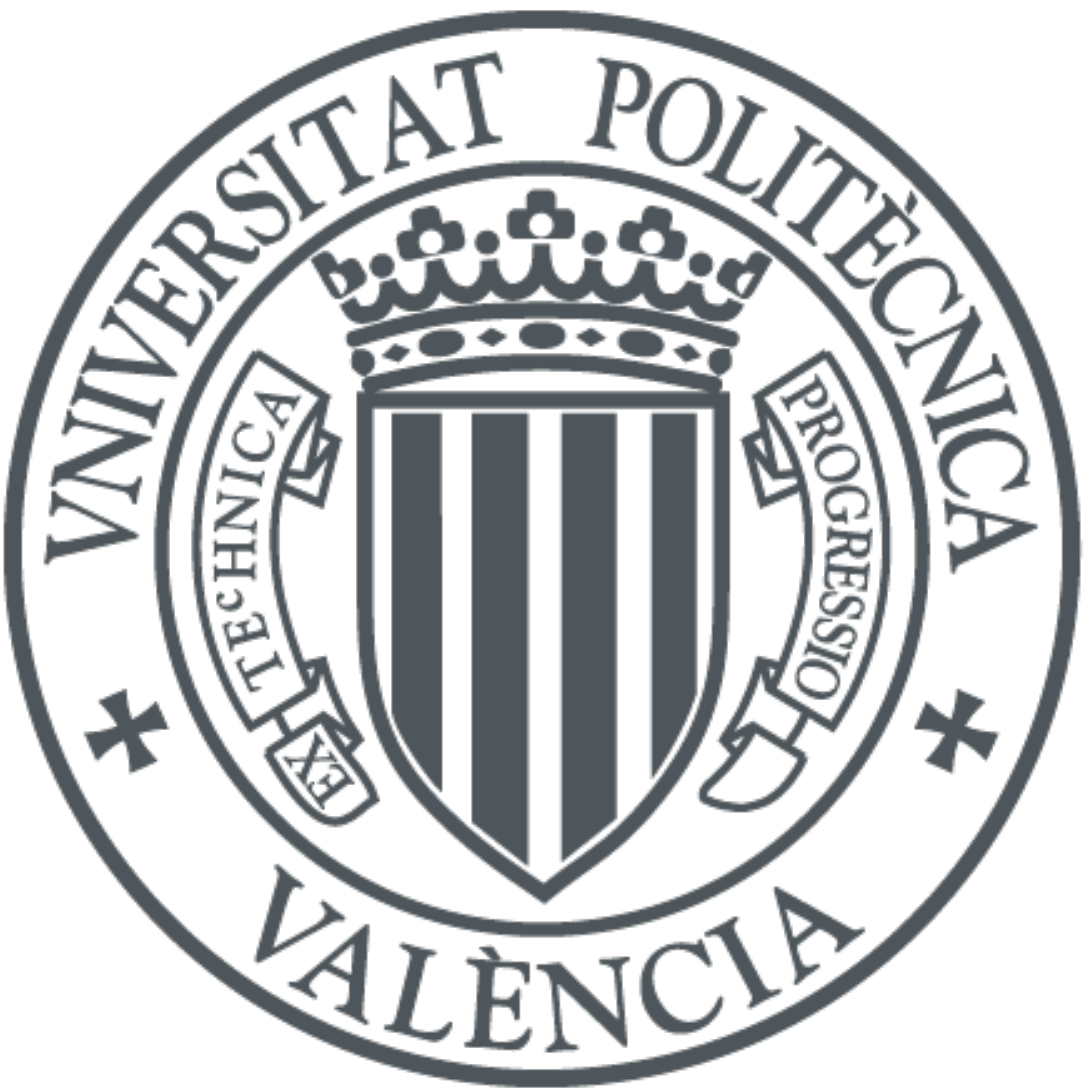

The final publication is available at

http://dx.doi.org/10.1016/j.pnucene.2011.07.009

Copyright Elsevier

Additional Information 


\title{
Performance of a transmutation advanced device for sustainable energy application
}

\author{
C. García ${ }^{\mathrm{a}, *}$, J. Rosales a , L. García ${ }^{\mathrm{a}}$, A. Pérez-Navarro ${ }^{c}$, A. Escrivác ${ }^{c}$, A. Abánades ${ }^{\mathrm{b}}$ \\ a Instituto Superior de Tecnologias y Ciencias Aplicadas (InsTEC), Avenida Salvador Allende y Luaces, Quinta de Los Molinos, Plaza, La Habana, Cuba \\ ${ }^{\mathrm{b}}$ Grupo de Modelización de Sistemas Termoenergéticos, Universidad Politécnica de Madrid, c/Ramiro de Maeztu 7, 28040 Madrid, Spain \\ ${ }^{\mathrm{c}}$ Instituto de Ingeniería Energética, Universidad Politécnica de Valencia, Valencia 46022, Spain
}

Keywords:

Very high temperature reactor

Nuclear waste

Transmutation

\begin{abstract}
A B S T R A C T
Preliminary studies have been performed to design a device for nuclear waste transmutation and hydrogen generation based on a gas-cooled pebble bed accelerator driven system, TADSEA (Transmutation Advanced Device for Sustainable Energy Application). In previous studies we have addressed the viability of an ADS Transmutation device that uses as fuel wastes from the existing LWR power plants, encapsulated in graphite in the form of pebble beds, cooled by helium which enables high temperatures (in the order of $1200 \mathrm{~K}$ ), to generate hydrogen from water either by high temperature electrolysis or by thermochemical cycles. For designing this device several configurations were studied, including several reflectors thickness, to achieve the desired parameters, the transmutation of nuclear waste and the production of 100 MW of thermal power. In this paper new studies performed on deep burn in-core fuel management strategy for LWR waste are presented. The fuel cycle on TADSEA device has been analyzed based on both: driven and transmutation fuel that had been proposed by the General Atomic design of a gas turbine-modular helium reactor. The transmutation results of the three fuel management strategies, using driven, transmutation and standard LWR spent fuel were compared, and several parameters describing the neutron performance of TADSEA nuclear core as the fuel and moderator temperature reactivity coefficients and transmutation chain, are also presented.
\end{abstract}

\section{Introduction}

Nuclear energy sustainability will depend on the actual capability of reducing both the inventory and long-term radiotoxicity of nuclear waste of present fission reactors, mainly dominated by the amount of transuranic isotopes remaining on the spent fuel. The IV Nuclear Energy Reactors Generation and the accelerator driven systems (ADS) are the two main options to achieve this goal. Preliminary studies have been made to design a device for simultaneous transmutation of nuclear waste and hydrogen generation, based on an ADS that uses the fuel containing the wastes from the existing LWR power plants, encapsulated in graphite, in the form of pebble beds, cooled by helium (Abánades et al., 2011), called TADSEA (Transmutation Advanced Device for Sustainable Energy Application) which enables high temperatures, in the order of $1200 \mathrm{~K}$ and $100 \mathrm{MW}$ of thermal power, to facilitate hydrogen generation either by high temperature electrolysis or by thermochemical cycles. Previously, in Abánades and Pérez-Navarro (2007), several studies were made to get an engineering design of the pebble bed transmuter (PBT), that contributed to the TADSEA's design. Similarly, PBT is a gas-cooled subcritical nuclear core, filled with graphite- fuel pebbles and driven by a cyclotron accelerator. PBT uses the well known TRISO fuel (U.S. NUREG, 2004). It can work based on the deep burn concept and the initial fuel loaded in PBT includes plutonium and minor actinides isotopes in a proportion similar to the existing in the typical spent fuel from a LWR power plant with a burnup of $40 \mathrm{MWd} / \mathrm{kg}$ and after 15 years of radioactive cooling in the plant pools (The European Technical Working Group on ADS, 2001).

In this paper new studies about deep burn strategies of spent fuel from LWR on TADSEA and PBT are presented. The behavior in PBT of the spent fuel management strategy, proposed by (Tálamo et al., 2004, Rodríguez et al., 2003) for the so called Deep Burn Modular Helium Reactor (DB-MHR) are analyzed. Three strategies for fuel management are compared: first of all, the traditional one, named "spent fuel"(SF) that uses spent fuel from LWR Second, the one in which the transuranic elements are divided in two groups, a "Driven Fuel" (DF) composed only by Plutonium isotopes and 
$\mathrm{Np}^{237}$ is used in PBT core like SF, and third, the fuel management strategy in which after the DF has reached the stationary state we feed PBT core with a layer of "Transmutation Fuel" (TF), composed by $\mathrm{Am}^{241}, \mathrm{Am}^{243}$ and $\mathrm{Cm}^{244}$ isotopes from spent $\mathrm{DF}$ and it is mixed after discharge from the reactor core with the Am and $\mathrm{Cm}$ isotopes, which were set-aside after UREX process (Laidler et al., 2001).

Mass depletion of Plutonium isotope and Minor Actinides (MA) after an irradiation of 990 days in the system, and the time evolution of radiotoxicity for the initial load and the unload fuel of the three strategies of fuel irradiation are compared.

For a better understanding of this process, a transmutation chain for TF obtained by MCNPX code, was built. It describes how the isotopic changes of main transuranic isotope occur. We also show parameters describing the neutronic behavior of TADSEA, such as the fuel and moderator temperature reactivity coefficients.

\section{Main PBT and TADSEA characteristics}

\subsection{PBT characteristics}

PBT is a subcritical system cooled by helium and moderated with graphite that uses as fuel small amounts of transuranics elements diluted in the form of TRISO particles in a graphite matrix, as to form a pebble bed configuration as displayed at Fig. 1. The system is maintained by a proton accelerator with a beam energy in the order of $380 \mathrm{MeV}$ and intensities close to $10 \mathrm{~mA}$. The spallation target that allocates a eutectic lead-bismuth in molten state uses a new geometrical design in a conical form to alleviate stress in the window and increase the neutron source size. Core of the assembly is formed by a cylinder containing the pebbles and, in its center, the spallation target. Fuel is confined in $3 \mathrm{~cm}$ radius pebbles, with an external layer of $5 \mathrm{~mm}$ thickness in pyrolytic graphite and the remaining sphere of $2.5 \mathrm{~cm}$ filled up with TRISO particles of $1 \mathrm{~mm}$ diameter containing the fuel in a structure that includes an external isotropic pyrolytic graphite layer, a SiC barrier, an inner isotropic pyrolytic graphite layer and, in the center, a buffer composed by a porous pyrolytic graphite.

The main advantage of a pebble bed core is the possibility for a continuous core recharge without plant operation interruption. Besides, the porous material in the TRISO particles facilitates the storage of big quantities of fission products and enables the system to reach higher burnups, in the order of $740 \mathrm{MWd} / \mathrm{Kg}$ (Rodríguez et al., 2003) and can afford a temperature of $1600{ }^{\circ} \mathrm{C}$. In a repository environment, spent TRISO particles should maintain their

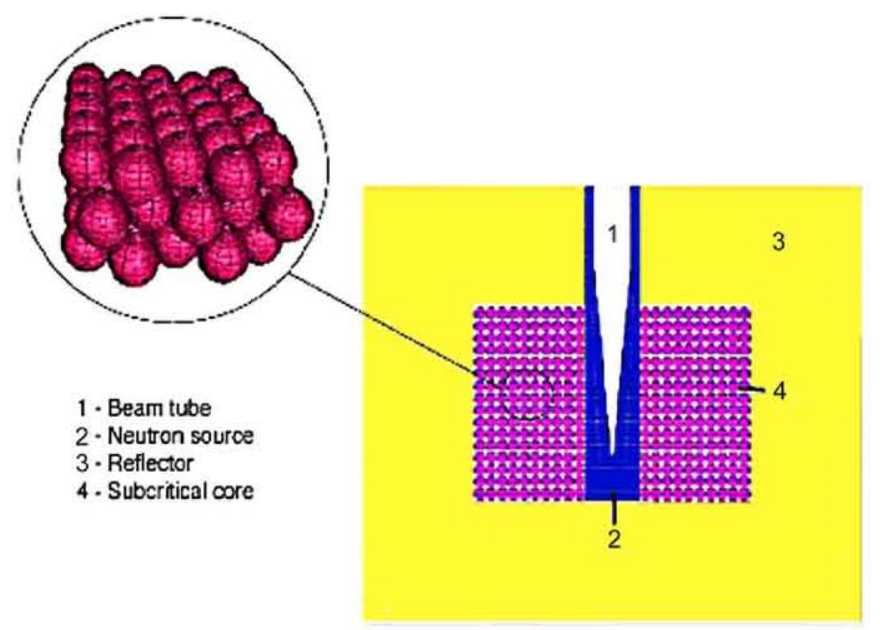

Fig. 1. PBT configuration. integrity for millions of years, even if they would permanently be flooded with groundwater.

PBT core is composed by 20 layers of pebbles disposed in a honeycomb way (hexagonal lattice), the pebbles stand over the holes of the layer immediately below, the packing factor is in the order of 0.74 and the thermal power is $10 \mathrm{MW}$ in the stationary state.

\subsection{TADSEA characteristics}

For the application to hydrogen generation, given the big amounts of this energy vector required in a future energy scenario, it was important to increase as much as possible the TADSEA thermal power looking for values in the order of $100 \mathrm{MW}$. With this goal, the proton beam characteristics have been increased up to the limits expected in the technical development of accelerators in the near future: $1 \mathrm{GeV}$ and $10 \mathrm{~mA}$. Besides, increasing the geometrical dimensions and maintaining the maximum power density in a value $\left(7 \mathrm{~W} / \mathrm{cm}^{3}\right)$, acceptable from a technical point of view, it is also possible to increase power, but with the constraint of keeping the transmutation capabilities of the system and no deterioration of the power profiles.

To guarantee the safety of the device, a study on the dependence of the multiplication factor on the reflector thickness was addressed. Results have indicated that for the most extreme condition, reflector radial thickness below $70 \mathrm{~cm}$ guarantees Keff always below 0.95 . Table 1 summarises the main parameters for both, PBT and TADSEA devices.

\subsection{Neutronics calculations}

For simulation purposes, the PBT and TADSEA cores were divided in 10 horizontal layers that were considered homogeneous zones in the calculations. The PBT and TADSEA neutronics were calculated with MCNPX 2.6e. The MCNPX 2.6e code (Gregg McKinney et al., 2007) has been chosen to simulate the neutronics behavior, because this new version incorporates a set of capabilities to facilitate the simulation of ADS transmutation systems, such as the LAHET code (Prael and Lichtenstein, 1989) that allows for the calculation of the neutron chain generated by a proton beam and its transport at high energies, making possible to simulate, in a single run, the transport of protons and neutrons in the spallation target and the incorporation of the neutrons, as a fast neutrons source, to the core of the subcritical assembly containing the fuel based on the nuclear wastes.

The new version also incorporates the CINDER90 and the MonteBurns codes that facilitate the calculation of the isotopes evolution in the core. Currently, the MC-NPX's possibilities to calculate the fuel burnup and the variation in the isotope composition are limited to critical configurations; therefore in our

Table 1

Main parameters of PBT and TADSEA.

\begin{tabular}{lll}
\hline Parameter & Value & \\
\hline Thermal power & $10 \mathrm{Mw}$ & $100 \mathrm{Mw}$ \\
Accelerator power & $3.8 \mathrm{Mw}$ & $10 \mathrm{Mw}$ \\
Proton beam energy & $380 \mathrm{Mev}$ & $1000 \mathrm{Mev}$ \\
Proton beam intensity & $10 \mathrm{~mA}$ & $10 \mathrm{~mA}$ \\
Fuel & Balls with & Balls with \\
& graphite + TRUs & graphite + TRUs \\
Fuel mass (Pu + MA) & $14.64 \mathrm{~kg}$ & $124.5 \mathrm{~kg}$ \\
Core volume & $1.69 \mathrm{~m}^{3}$ & $14.38 \mathrm{~m}^{3}$ \\
Number of balls & 11064 & 94092 \\
Average power density & $6 \mathrm{~W} / \mathrm{cm}^{3}$ & $7 \mathrm{~W} / \mathrm{cm}^{3}$ \\
Keff & 0.84 & 0.94 \\
\hline
\end{tabular}


simulations the burnup calculations in the subcritical core were made considering an average neutron flux in each zone condensed to a single speed and spatially averaging for the flux distribution deduced from the eigenvalues problem analysis. CIN-DER90 works with 63 energy groups, and the transversal cross sections for these 63 groups are condensed using a generic spectrum. This approach can produce some discrepancies in the final inventory for the isotopes generated from the fission products. Nevertheless, for the comparison of different configurations this limitation does not introduce significant errors, as it was demonstrated before (García et al, in press). Another MCNPX.2.6e new capability used for this study is the incorporation of the predictor-corrector technique for the burnup calculation. This technique allows for a use of longer time step without significant precision loss in the results.

\section{Spent fuel management strategies for PBT and TADSEA}

Preliminary studies have shown that the isotopic depletion of nuclear fuel under deep burn concept in PBT and TADSEA subcritical cores has a similar behaviour. Therefore, it was decided to compare three different spent fuel management strategies, called "spent fuel" (SF), "driven fuel"(DF) and DF + SF, and to model the burnup using the PBT core as it demands a lower computational cost due to its smaller size.

\subsection{Fuel shuffling strategies}

The deep burn concept is based on the use of driven fuel, rich in fissile actinides $\left(\mathrm{Pu}^{239}\right)$ and transmutation fuel, rich in non-fissile actinides. The DF provides the excess reactivity to drive the power production and sustain large effective transmutation rates. The TF provides burnable poison and reactivity control. The LWRs waste is reprocessed by uranium and fission products extraction (UREX). The final products of the LWRs spent fuel reprocessing are NpPuO1.7 and AmCm01.7; the first material constitutes the driven fuel (DF) (Tálamo et al., 2004). The DF is the primary nuclear fuel for the TADSEA or PBT and it sustains the fission chain reaction, mainly by $\mathrm{Pu}^{239}$. AmCm01.7 from spent DF is mixed after discharge from the reactor core with the AmCm01.7, which were set-aside after UREX process, to build fresh TF. After irradiation, spent TF is sent into the repository.

The main objective of the DF concept has been the extended destruction of $\mathrm{Pu}^{239}$, one of the major contributors to long lasting radiotoxicity and the major proliferation concern. We have also focused our attention on the transmutation of MA because of their radiological importance, and we tried to get their transmutation process with the TF burning.

PBT operation procedure assumed for the simulation includes an initial step where each level is filled with fresh fuel. And in cycles of 99 days, each layer is moved to a lower level, introducing new fuel on the top layer and extracting the balls from the bottom one. With this scheme, the fuel is burned up during 990 days and, after 10 cycles; the system reaches composition equilibrium, where there is only fresh fuel in the first layer while for the last one the fuel has passed a complete 99 days cycle for each of the previous layers. We simulated three spent fuel management strategies in PBT core. In SF strategy the core is filled with fresh fuel from typical spent fuel from a LWR. DF fuel shuffling strategy is like SF. When DF reached the equilibrium stage (here we considered 20 cycles), we feed a layer with fresh TF, after we continued feeding with DF layers. The TF layer crosses the PBT core during 10 cycles, then it is extracted as irradiation fuel and sent into repository. The layer loaded with TF contains the AmCm01.7 mass, which was set-aside after UREX process when the DF was made, plus the AmCm01.7 masses
Table 2

Initial and final composition of SF in PBT core.

\begin{tabular}{lccl}
\hline Isotope & Initial mass $(\mathrm{g})$ & Final mass $(\mathrm{g})$ & \% \\
\hline $\mathrm{Np}^{237}$ & 655 & 251.1 & +61.6 \\
$\mathrm{Am}^{241}$ & 752 & 41.2 & +94.4 \\
$\mathrm{Cm}^{242}$ & 0 & 78.2 & - \\
$\mathrm{Am}^{243}$ & 134 & 485.8 & -262.5 \\
$\mathrm{Cm}^{244}$ & 24 & 427.2 & -1680 \\
$\mathrm{Pu}^{238}$ & 203.1 & 635.8 & -213 \\
$\mathrm{Pu}^{239}$ & 7512 & 117.6 & +98.4 \\
$\mathrm{Pu}^{240}$ & 3482 & 364.8 & +89.5 \\
$\mathrm{Pu}^{241}$ & 1165 & 409.3 & +64.9 \\
$\mathrm{Pu}^{242}$ & 713 & 1742 & -144.3 \\
\hline
\end{tabular}

belonging to 9 irradiated layers of DF that stood 990 days in the PBT core. The irradiated DF was reprocessed in a second step.

\subsection{Evolution of isotopic composition for studied spent fuel management strategies}

Tables 2 and 3 show the mass load and unload of PBT for SF and DF strategies. The fourth column gives the variation in \% of initial mass relative to final mass according to the following expression:

$\Delta \rho^{j}=\left\{\frac{\rho_{\text {initial }}^{j}-\rho_{\text {final }}^{j}}{\rho_{\text {initial }}^{j}}\right\} \times 100$

Where $\Delta \rho^{j}$ is the variation of mass of isotope $j$ with burnup. $\rho_{\text {initialfinal }}^{j}$ is the initial or final mass of isotope $j$.

The precedent + means decrease and precedent - means increase.

In Table 3, the values of mass variation (\%) of $\mathrm{Am}^{241}, \mathrm{Am}^{243}$ and $\mathrm{Cm}^{244}$ are in relationship to the masses which were set-aside after UREX process when was fresh DF building, because the initial masses of those isotopes in DF were zero.

We can observe in DF that masses of $\mathrm{Cm}^{242}, \mathrm{Am}^{243}$ and $\mathrm{Cm}^{244}$ increase, nevertheless they grow less than masses of the same isotopes in SF. Am ${ }^{241}$ in DF grows softly; it is logical because it is absent in the initial load. In Fig. 3 (b) we can see that $\mathrm{Am}^{241}$ mass reaches a peak and then begins to decrease. In SF, where $\mathrm{Am}^{241}$ has a big initial mass, it decreases considerably with burnup.

In both cases ( $S F$ and DF), the $\mathrm{Pu}^{239}$ mass and $\mathrm{Pu}^{240}$ mass afford a great depletion, it is the main goal of deep burn concept, while $\mathrm{Np}^{237}$ and $\mathrm{Pu}^{241}$ decrease their mass too, but $\mathrm{Pu}^{241}$ less in DF. The $\mathrm{Pu}^{238}$ mass grows considerably more in $\mathrm{SF}$ than $\mathrm{DF}$, and $\mathrm{Pu}^{242}$ mass grows softly less in DF (See Fig. 2 (a) and (b)).

The whole mass of Plutonium isotopes decreases with burning for DF in $72 \%$, while SF achieves $75 \%$. The difference is because DF is formed fundamentally by Plutonium obtaining the same burnup needs a longer cycle. Plutonium has a similar behavior with burnup for both spent fuel management strategies.

Table 3

Initial and final composition of DF in PBT core.

\begin{tabular}{lccl}
\hline Isotope & Initial mass $(\mathrm{g})$ & Final mass $(\mathrm{g})$ & \% \\
\hline $\mathrm{Np}^{237}$ & 759.1 & 363.3 & +52.1 \\
$\mathrm{Am}^{241}$ & 0 & 61.5 & -6.3 \\
$\mathrm{Cm}^{242}$ & 0 & 45.7 & - \\
$\mathrm{Am}^{243}$ & 0 & 204.8 & -213.9 \\
$\mathrm{Cm}^{244}$ & 0 & 236 & -593 \\
$\mathrm{Pu}^{238}$ & 223.1 & 439.9 & -97.5 \\
$\mathrm{Pu}^{239}$ & 8469 & 208.7 & +97.5 \\
$\mathrm{Pu}^{240}$ & 3423 & 749.4 & +78.1 \\
$\mathrm{Pu}^{241}$ & 1251 & 948.8 & +24.2 \\
$\mathrm{Pu}^{242}$ & 759.1 & 1667.0 & -119.6 \\
\hline
\end{tabular}



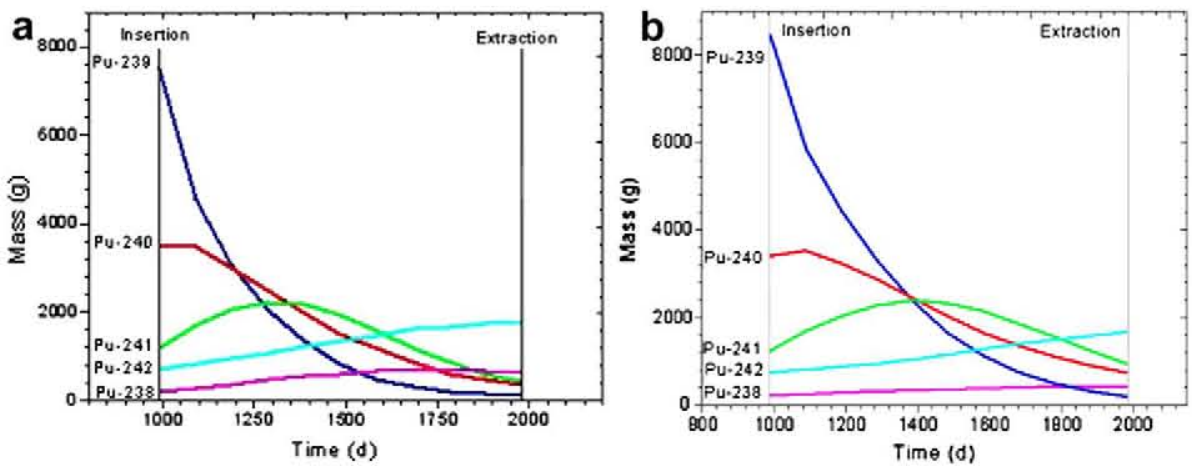

Fig. 2. Results for the evolution of Pu isotopes in PBT (a) SF, (b) DF.
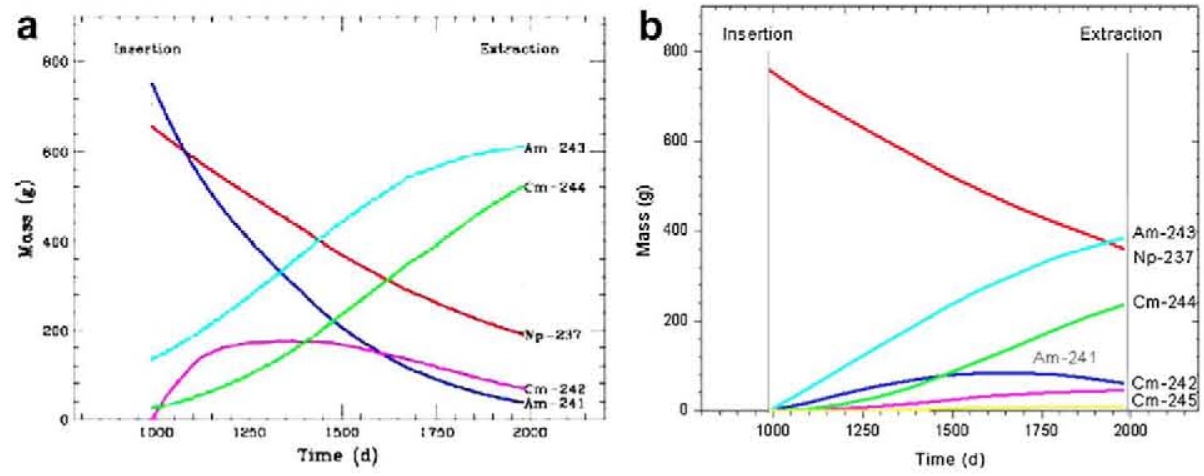

Fig. 3. Results for the evolution of MA isotopes in PBT (a) SF, (b) DF.

The whole depletion of MA ( $\mathrm{Np}, \mathrm{Am}$ and $\mathrm{Cm}$ ) with burnup is an $18 \%$ rate decreasing for $\mathrm{SF}$ and a $16 \%$ rate increasing for $\mathrm{DF}$, considering the mass which were set-aside after UREX process. The last takes place because DF is not formed by $\mathrm{Am}^{241}$. This isotope has a great depletion with burnup for SF: $\mathrm{Am}^{241}$ becomes to $\mathrm{Cm}^{242}$ by successive neutronics capture and beta disintegration. Due to there are not $\mathrm{Am}^{241}$ for initial composition of $\mathrm{DF}$, then $\mathrm{Pu}^{238}$ increases less than for SF.

When the stationary stage is reached for DF strategy we began a new fuel cycle: we feed PBT core with a fresh TF layer; then we continued feeding with DF layers. The spent fuel management strategy using one layer of TF and 9 layers of DF was called DF + TF strategy. Here the layer of TF crosses PBT core in 99 days cycles. Fig. 4 (a) and (b) shows the time variation of the mass for the different plutonium isotopes and the minor actinides, respectively, for the layer of TF crossing the PBT core. Table 4 shows the initial and final masses of MA and Plutonium isotopes for 990 days of burning of the TF layer. The variation (\%) is referred to initial mass of MA only. In Table 5 we can see initial and final masses of transuranic for the DF + TF strategy.

In Table 4 we can observe that among the Plutonium isotopes, only $\mathrm{Pu}^{238}$ has a significant buildup in TF layer. It reaches a stationary concentration because the appearing process $\left(\mathrm{Cm}^{242}\right.$ disintegration) and disappearing process (neutronics capture), reaching the equilibrium. The DF + TF strategy (Table 5), we can see that initial mass of $\mathrm{Pu}^{238}$ increases $289 \%$; it is higher than for SF (213\%). Nevertheless $\mathrm{Am}^{243}$ mass decreases significantly, while in SF enlarges.

The $\mathrm{Am}^{241}$ mass decreases a $96.5 \%$ in the DF + TF strategy, while decreases a $94.5 \%$ for $\mathrm{SF}$. The $\mathrm{Cm}^{244}$ mass increases too for the
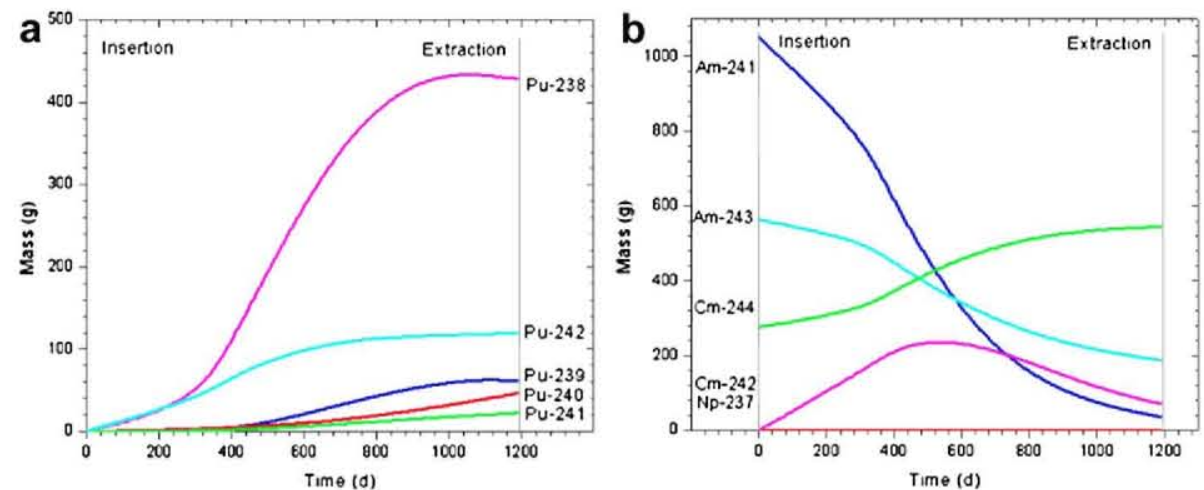

Fig. 4. Results for the evolution of Pu (a) and MA (b) isotopes in TF layers of PBT. 
Table 4

Initial and final composition of TF in PBT core $(*)$ The variation in $\%$ is referred to initial mass of MA.

\begin{tabular}{lccl}
\hline Isotope & Initial mass $(\mathrm{g})$ & Final mass $(\mathrm{g})$ & \% Depletion \\
\hline $\mathrm{Np}^{237}$ & 0.0 & 0.8 & $0.04\left(^{*}\right)$ \\
$\mathrm{Am}^{241}$ & 1050 & 36.5 & +96.5 \\
$\mathrm{Cm}^{242}$ & 0.0 & 71 & $3.7\left(^{*}\right)$ \\
$\mathrm{Am}^{243}$ & 563.8 & 188.2 & +66.6 \\
$\mathrm{Cm}^{244}$ & 275.9 & 545.2 & -97.6 \\
$\mathrm{Pu}^{238}$ & 0.0 & 428.3 & $22.7\left(^{*}\right)$ \\
$\mathrm{Pu}^{239}$ & 0.0 & 61.5 & $3.3\left(^{*}\right)$ \\
$\mathrm{Pu}^{240}$ & 0.0 & 46 & $2.4\left(^{*}\right)$ \\
$\mathrm{Pu}^{241}$ & 0.0 & 22.2 & $1.2\left(^{*}\right)$ \\
$\mathrm{Pu}^{242}$ & 0.0 & 119.7 & $6.3\left(^{*}\right)$ \\
\hline
\end{tabular}

Table 5

Initial and final composition of DF $+\mathrm{TF}$ in PBT core.

\begin{tabular}{lccl}
\hline Isotope & Initial mass $(\mathrm{g})$ & Final mass $(\mathrm{g})$ & \%Depletion \\
\hline $\mathrm{Np}^{237}$ & 759.1 & 364.1 & +52 \\
$\mathrm{Am}^{241}$ & 1050 & 36.5 & +96.5 \\
$\mathrm{Cm}^{242}$ & 0.0 & 116.7 & - \\
$\mathrm{Am}^{243}$ & 563.8 & 188.2 & +66.6 \\
$\mathrm{Cm}^{244}$ & 275.9 & 545.2 & -97.6 \\
$\mathrm{Pu}^{238}$ & 223 & 868.2 & -289.3 \\
$\mathrm{Pu}^{239}$ & 8469 & 270.2 & +96.8 \\
$\mathrm{Pu}^{240}$ & 3423 & 795.4 & +76.8 \\
$\mathrm{Pu}^{241}$ & 1251 & 971 & +22.4 \\
$\mathrm{Pu}^{242}$ & 759.1 & 1786.7 & -135.4 \\
\hline
\end{tabular}

DF + TF strategy but increases less than for SF (96.7\% and 1680\% respectively). The last behavior occurs mainly caused by $\mathrm{Cm}^{244}$, which comes from quickly disintegration of $\mathrm{Am}^{244}$ and accumulates more for SF, because here more $\mathrm{Am}^{243}$ exists. (see Fig. 3 (a)).

Summarizing, when compared with the SF strategy, the DF + TF strategy obtained a significant decrease of $\mathrm{Am}^{243}$ initial mass, more decrease in the $\mathrm{Am}^{241}$ mass, less rise of $\mathrm{Cm}^{244}$, and a buildup of $\mathrm{Pu}^{238}$; it also maintained the same reduction in $\mathrm{Np}^{237}$ and the rest of Plutonium isotopes, except $\mathrm{Pu}^{241}$.

\subsection{Transmutation chain}

We calculated the one speed capture and fission cross sections of transuranic elements that they form the Transmutation Fuel and we built the transmutation chain for it.

The transmutation chain shows disintegration, capture and fission relative probabilities of each isotope of the chain; it allows explaining their transmutation probability under specific operation PBT conditions.

Cross sections were calculated for the end of each 99 days cycle, for each interesting transuranic isotope.

In all cases we obtained similar results; because of that we only show the values at the end of the first cycle. Fig. 5 presents the transmutation chain for burning TF, at the end of the first cycle, or first layer at 99 days of burning. Results were compared with those obtained by (Talamo et al., 2004) and we observed a very good agreement.

\subsection{Time evolution of radiotoxicity for the studied spent fuel management strategies}

The radiotoxicity of a nuclide is determined by the product of the activity and the effective dose coefficient $e$ for a given isotope Radiotoxicity $=$ Activity $\times e$.

The activity is just the number of disintegrations per second and is measured in Becquerel units, $\mathrm{Bq}(1 \mathrm{~Bq}=1$ disintegration per second). The effective dose coefficient $e$ is a measure of the damage caused by ionising radiation associated with the radioactivity of an isotope. It accounts for radiation and tissue weighting factors, metabolic and biokinetic information. It is measured in Sievert per

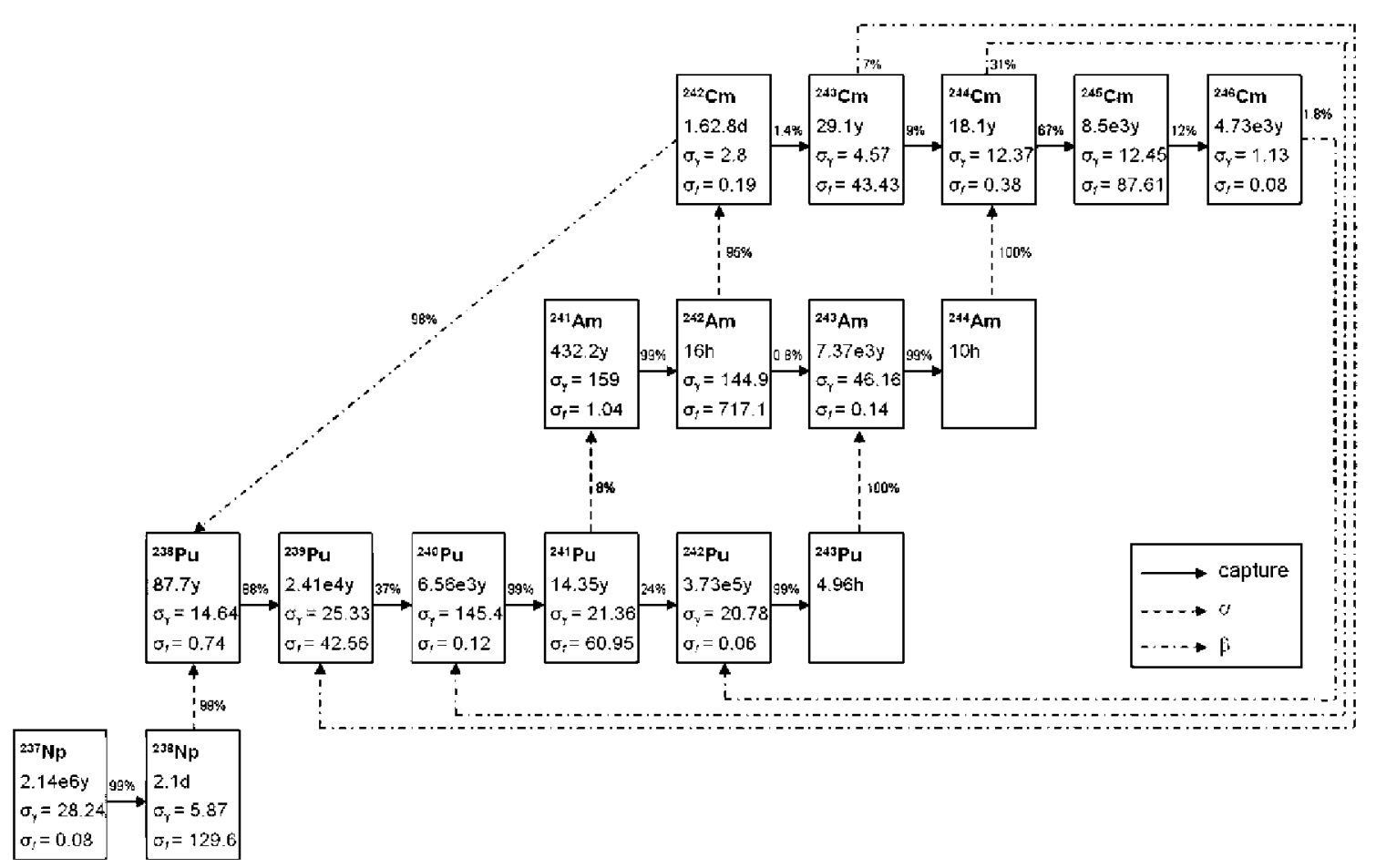

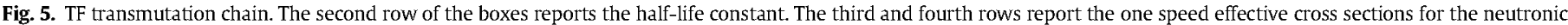

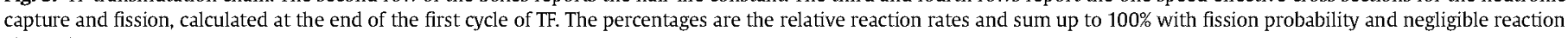
channels. 
Becquerel $(\mathrm{Sv} / \mathrm{Bq})$ units, where the Sievert is a measure of the dose arising from the ionisation energy absorbed (The European Technical Working Group on ADS, 2001).

In calculating radiotoxicity, normalized to the initial mass of the fuel loaded in the device, we have taken into account both the initial isotopes and those with significant lifetimes generated along the cycle.
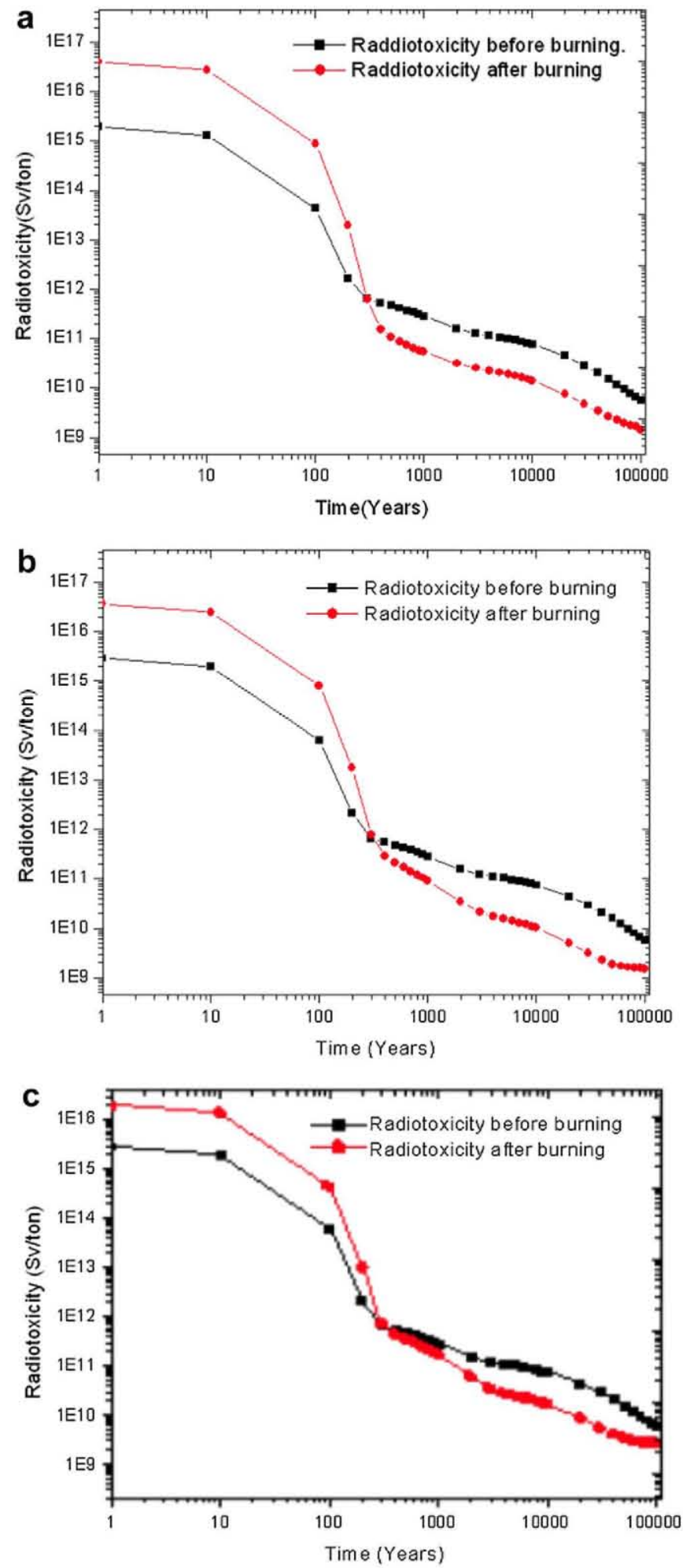

Fig. 6. Time evolution of radiotoxicity for SF (a), DF $+\mathrm{TF}(\mathrm{b})$ and $\mathrm{DF}(\mathrm{c})$.
Fig. 6 (a), (b) and (c) shows the time evolution of radiotoxicity by inhalation for both: the charge and the discharge of system for spent fuel management strategies $\mathrm{SF}$, the $\mathrm{DF}+\mathrm{TF}$ and $\mathrm{DF}$ respectively. In DF we considered the contribution to radiotoxicity for both: Am and $\mathrm{Cm}$ which were set-aside after UREX process.

In Fig. 6 (a) we can observe during the first 300 years there is a relative increase in radiotoxicity to discharge in relation to load. The last is possible because $\mathrm{Cm}^{244}$ appeared with fuel burning process and it has a half-life equal to 18 years and it contributes meaningfully to radiotoxicity in this period. But after this time the tendency reverts and an order of magnitude decrease is obtained for the discharge radiotoxicity in relation to the initial load one. For example, for 1000 years we obtained for $\mathrm{SF}\left(3.06 \times 10^{11}\right.$ to load, and $5.84 \times 10^{10} \mathrm{~Sv} /$ Ton, to unload).

In Fig. 6 (b) (DF + TF), we can see the same than for SF during the first 300 years. And here the tendency reverts too before this time. For longer time, before 1000 years the main contributions to load radiotoxicity are both: $\mathrm{Am}^{241}$ by its great values of activity and $\mathrm{Pu}^{240}$ by its big mass and great activity. The radiotoxicity values of both are an order of magnitude higher than remaining isotopes.

After 1000 years, the radiotoxicity time evolution has a similar behavior for SF and DF $+\mathrm{TF}$, although is slightly less for DF $+\mathrm{TF}$. For example, for 5000 years for SF and DF + TF the radiotoxicity values to load are $1.06 \times 10^{11} \mathrm{~Sv} /$ ton, and $1.02 \times 10^{11} \mathrm{~Sv} /$ ton respectively, and to unload are $2.07 \times 10^{10} \mathrm{~Sv} /$ ton and $1.53 \times 10^{10} \mathrm{~Sv} / \mathrm{ton}, 35 \%$ less for $D F+T F$ to unload. In this time the main contributions to radiotoxicity to load are from the $\mathrm{Pu}^{239}$ and $\mathrm{Pu}^{240}$, and to unload from the $\mathrm{Pu}^{240}$.

For longer time, the radiotoxicity to unload for $\mathrm{DF}+\mathrm{TF}$ will be always less than $\mathrm{SF}$, for example, at 10,000 years for $\mathrm{DF}+\mathrm{TF}$ radiotoxicity is $1.01 \times 10^{10} \mathrm{~Sv} /$ ton and for SF is $1.42 \times 10^{10} \mathrm{~Sv} /$ ton $(40 \%$ less).

DF radiotoxicity to load (Fig. 6 (c)) has a similar behavior to the SF, and to unload it shows a light increase for time after 300 years in relation to $\mathrm{SF}$ and $\mathrm{DF}+\mathrm{TF}$.

After 300 years, spent fuel management strategy called DF $+\mathrm{TF}$ leads to a considerable decrease of the radiotoxicity time evolution in relation to the initial load one. This decreasing is stronger than for SF strategy in a 30-40\%. But if we take into account the poor energetic contribution of the TF layer and the need of a second step in reprocessing process, it is necessary to carry out a careful evaluation of the alternative of burning MA in a fast system with full recycling, and use the spent fuel management strategy called DF to obtain the main goal, the extended destruction of $\mathrm{Pu}^{239}$.

\section{Reactivity temperature coefficients}

We calculated the temperature coefficients for a temperature excursion affecting only the cross-sections either for the fuel or the moderator. Here we focused our studies at the beginning of the first cycle at PBT for DF composition. We considered that graphite inside pebbled matrix and metal fuel formed by $\mathrm{Np}^{237}$

Table 6

Fuel Temperature Reactivity Coefficients (FTRC).

\begin{tabular}{llll}
\hline & \multicolumn{2}{l}{ Temperature range $(\mathrm{K})$} \\
\cline { 2 - 4 } & $293.6-600$ & $600-800$ & $800-1200$ \\
\hline Keff $^{\mathrm{T}_{1}}$ & 0.90417 & 0.89588 & 0.88800 \\
Std desv. & 0.00051 & 0.00088 & 00089 \\
Keff $^{\mathrm{T}_{2}}$ & 0.89588 & 0.88800 & 0.88240 \\
Std dev. & 0.000888 & 0.00089 & 0.00085 \\
FTRC pcm/K & $-3.34 \pm 0.56$ & $-4.95 \pm 1.11$ & $-1.79 \pm 0.55$ \\
\hline
\end{tabular}




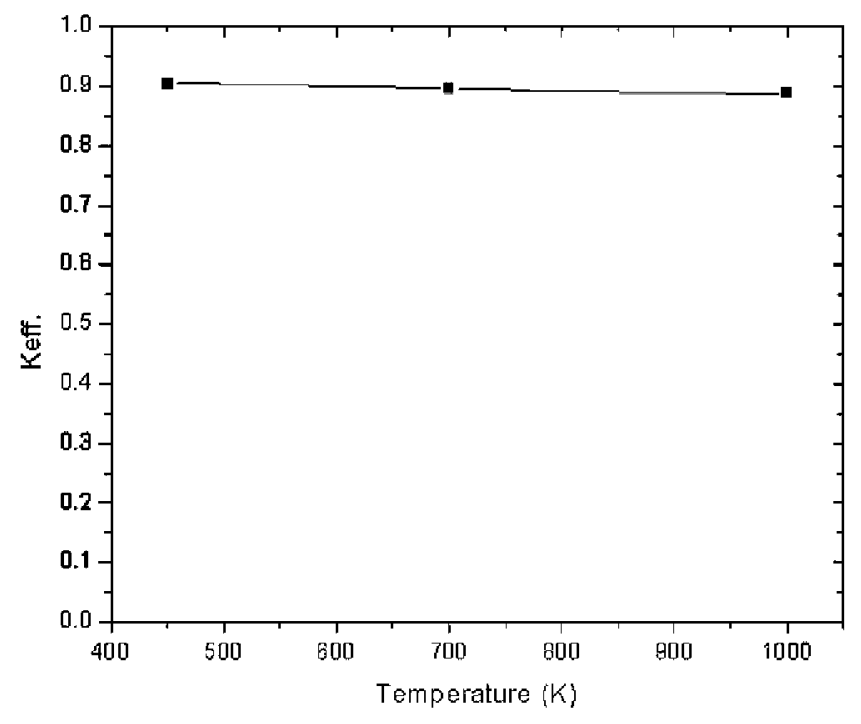

Fig. 7. Keff as function of fuel temperature in PBT, beginning of first cycle.

and Plutonium isotopes were at the same temperature. We used the available library in XSDIR, ENDL/B VI.2 (LA-CP-05-0369, 2005). We ran one thousand cycles and one thousand histories per cycle for eigenvalues calculations. Table 6 gives eigenvalues calculated for each fuel extreme temperature in the considered ranges and standard deviations too and the fuel temperature reactivity coefficients (FTRC), calculated by the following expression:

$F T C R=\frac{\rho^{T_{2}}-\rho^{T_{1}}}{T_{2}-T_{1}}=\frac{\frac{1}{K e f f T_{1}}-\frac{1}{K_{e f f T_{2}}}}{T_{2}-T_{1}}$

Where $\operatorname{Keff}^{T_{1}}$ and $\operatorname{Keff}^{T_{2}}$ are the eigenvalues at temperatures $T_{1}$ and $T_{2}$ respectively.

We obtained similar values to those reported by Zakova and Tálamo (2008) for Plutonium fuel. Fig. 7 shows the dependence of eigenvalue Keff on fuel temperature. We can observe that Keff decreases with temperature leading to a negative fuel temperature feedback.

Increasing the graphite temperature causes a shift to higher energies of the Maxwellian part of the neutron spectrum; this phenomenon drives the moderator temperature reactivity coefficient, especially when we have fuel with plutonium.

Table 7 shows the values of moderator temperature reactivity coefficients (MTRC) for several temperature ranges. We considered the same temperature at inside and outside graphite pebbled. For graphite of reflector, always we considered $300 \mathrm{~K}$ of temperature.

The lattice nuclei bindings are taken into account by the scattering function, $S(a, \mathrm{ft})$ of graphite, available in the ENDL/B V nuclear data library at $300,600,800,1200,1600$ and $2000 \mathrm{~K}$.

Table 7

Moderator Temperature Reactivity Coefficients (MTRC).

\begin{tabular}{llllll}
\hline $\begin{array}{l}\text { Temperature } \\
\text { range }(\mathrm{K})\end{array}$ & Keff $^{\mathrm{T}_{1}}$ & Std desv. & Keff $^{\mathrm{T}_{2}}$ & Std desv. & MTRC pcm/K \\
\hline $300-600$ & 0.91465 & 0.0006 & 0.90500 & 0.00062 & $-3.89+0.49$ \\
$600-800$ & 0.90500 & 0.00062 & 0.98170 & 0.00061 & $-8.24+0.76$ \\
$800-1200$ & 0.89170 & 0.00061 & 0.88143 & 0.00060 & $-3.27+0.38$ \\
$1200-1600$ & 0.88143 & 0.00060 & 0.85662 & 0.00058 & $-8.21+0.39$ \\
$1600-2000$ & 0.85662 & 0.00058 & 0.82606 & 0.00058 & $-10.80+0.41$ \\
\hline
\end{tabular}

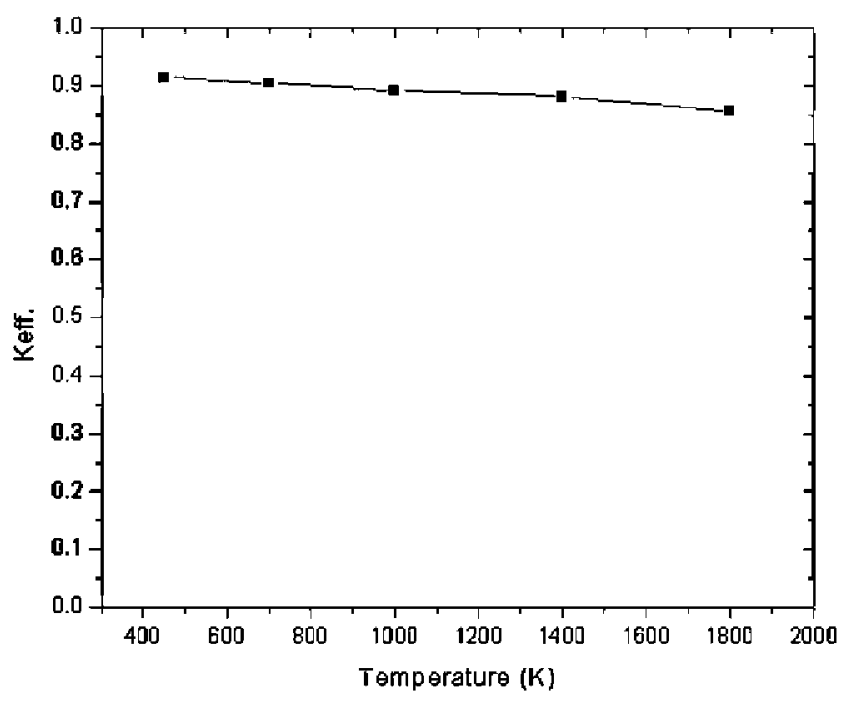

Fig. 8. Keff as function of moderator temperature in PBT, beginning of first cycle.

The MTRC values were calculated by similar expression that was used for FTRC. The MTRC values calculated by us for DF in PBT system have a good agreement with those reported by Zakova and Tálamo (2008) for plutonium fuel.

Fig. 8 shows the Keff dependence in PBT as function of the moderator temperature. We obtain a relatively more flat behavior of Keff as function of the temperature than the one reported for the advanced high temperature reactor (AHTR) proposed by the Oak Ridge National Laboratory, although we can watch here too that Keff curve undergoes a change of slope after $1200 \mathrm{~K}$, because the right part of the peak of the shifted spectrum covers the region around $1.056 \mathrm{eV}$ (at $1200-1500 \mathrm{~K}$ ), where the wide resolved resonance of $\mathrm{Pu}^{240}$ is located.

\section{Conclusions}

The present work is based on the pre-conceptual PBT and TADSEA designs and it focuses on the comparison of the behavior of several deep burn in-core fuel management strategies for LWR waste.

We analyzed the fuel cycle on TADSEA device based on driven and transmutation fuel that was proposed previously for the general atomic design of a gas turbine-modular helium reactor.

We compared the spent fuel management strategies from LWR waste in PBT system called SF, DF and DF $+\mathrm{TF}$, taking into account the decreasing of plutonium mass isotopes and minor actinides in relation to the initial load one, and decreasing of radiotoxicity time evolution of fuel to discharge.

The results of burning for SF and DF strategies in PBT and TADSEA at stationary stage lead to a similar reduction of masses of $\mathrm{Np}^{237}, \mathrm{Pu}^{239}$ and the $\mathrm{Pu}^{240}$, to a less increase for DF of masses of $\mathrm{Cm}^{242}, \mathrm{Cm}^{244}, \mathrm{Am}^{243}$ and $\mathrm{Pu}^{242}$, and how unfavorable effects a slight increase of $\mathrm{Am}^{241}$ for DF (it is obtained for SF a significant decrease) and a less decrease of $\mathrm{Pu}^{241}$.The time evolution of radiotoxicity to discharge for both is the same.

The spent fuel management strategy called DF + TF needs a two step fuel cycle, but it achieves a similar decrease of mass of $\mathrm{Am}^{241}$ like SF, a decrease of the mass of $\mathrm{Am}^{243}$ in a 66\% (grow for SF) and a less increase of $\mathrm{Cm}^{244}$, but the mass of $\mathrm{Pu}^{238}$ increases. After 300 years radiotoxicity to discharge of DF + TF strategy is 30 or $40 \%$ less than SF strategy.

But if we take into account the poor energetic contribution of the TF layer and the need of a second step in the reprocessing 
process, it is necessary to make a careful evaluation of the alternative of burning MA in a fast system with full recycling, and use the spent fuel management strategy called DF to obtain the main goal, the extended destruction of $\mathrm{Pu}^{239}$.

In this paper we also calculated the fuel and moderator reactivity temperature coefficients for the beginning of the first cycle for DF in PBT system. We obtained a very good agreement with those obtained before for AHTR using Plutonium fuel.

\section{Acknowledgements}

One of the authors (C Garcia) thanks Universidad Politecnia de Valencia for the support received from its INNOVA programme to finance his stay at the IIE to complete this study.

\section{References}

Abănades, A., García, C., García, L., Escrivâ, A., Pérez-Navarro, A., Rosales, J., 2011. Application of gas-cooled Accelerator Driven System (ADS) transmutation devices to sustainable energy development. Nuclear Engineering and Design $241,2288-2294$.

Abádanes, A., Perez-Navarro, A., 2007. Engineering design studies for transmutation of nuclear waste with a gas-cooled pebble-bed ADS. Nuclear Engineering and Design 237, 325-333.
García, C., Pérez-Navarro, A., Escriva, A., Abanades, A., Rosales, J., Garcia, L. Autonomous hydrogen generator based on an ADS transmutation system, in press.

Gregg McKinney, et al., 2007. MCNPX 2.6x Features (20062007). LA-UR-07-2053. Los Alamos National Laboratory.

LA-CP-05-0369, April 2005. MCNPX Users Manual Version 2.5.0.

Laidler, James J., Burris, Leslie, Coll1ns, Emory D., Duguid, James, Henry, Roger N. Hill, Julian, Karell, Eric J., Mcdeavitt, Sean M., Thompson, Major Williamson, Mark A., Willit, James L., 2001. Chemical partitioning technologies for an ATW system. Progress in Nuclear Energy 38 (No. I-2), 65-79.

Prael, R.E., Lichtenstein, H., 1989.Use guide to LCS: The LAHET Code System, Group X-6. MSB226. Los Alamos National Laboratory.

Rodríguez, C., Baxter, A., McEachern, D., Fikani, M., Venneri, F., 2003. Deep-Burn making nuclear waste transmutation practical. Nuclear Engineering and Design 222, 299-317.

Tálamo, Alberto, Gudowski, Waclaw, Venneri, Francesco, 2004. The burnup capabilities of the deep burn modular helium reactor analyzed by the Monte Carlo continuous energy code MCB. Annals of Nuclear Energy 31, $173-196$

The European Technical Working Group on ADS, April 2001. A European Roadmap for Developing Accelerator Driven Systems for Nuclear Waste Incineration.

U.S. NUREG, Nuclear Regulatory Commission, 2004. TRISO - Coated Particle Fue Phenomenon Identification and Ranking Tables (PIRTs) for Fission Product Transport Due to Manufacturing, Operations, and Accidents. Office of Nuclear Regulatory Research; NUREG/CR - 6844, Vol. 1.

Zakova, Jitka, Talamo, Alberto, 2008. Analysis of reactivity coefficients of advanced high-temperature for plutonium and uranium fuels. Annals of Nuclear Energy $35,904-916$ 